\title{
Forschung in Digitalen Innovationsprojekten - zwischen Praxistauglichkeit und wissenschaftlicher Relevanz
}

\author{
Tim Scheplitz • Martin Benedict (D) Hannes Schlieter (D) • \\ Stefanie Kaczmarek $\cdot$ Marcel Susky
}

Eingegangen: 7. November 2019 / Angenommen: 13. Februar 2020 / Online publiziert: 25. Februar 2020 (C) Der/die Autor(en) 2020

Zusammenfassung Ergebnisse Digitaler Innovationsprojekte (DIP) sind zumeist neue Artefakte von Informationssystemen, welche stark an die Resultate gestaltungsorientierter Forschung erinnern - dem sogenannten Design Science. Dennoch halten die Erkenntnisse aus DIP der Praxis nicht hinreichend Einzug in die wissenschaftliche Gemeinschaft. So zum Beispiel im Gesundheitswesen. Hier werden zwar verstärkt DIP forciert, ihre zentralen Erkenntnisse zur erfolgreichen Gestaltung jedoch selten in die Community transportiert. Ebenso wenig haben sich abseits klassischer Publikationswege Standards zur Kommunikation von erarbeitetem Wissen etabliert. Explizites und implizites Wissen, als Projektresultate nebst der Innovation selbst, können für die Organisation und Durchführung von vergleichbaren Projekten von kritischer Bedeutung sein kann. Ihre Kommunikation und strukturierte Bereitstellung wurden bislang in der Wissenschaft wenig adressiert. Das Ziel dieses Beitrags ist daher einen Weg aufzuzeigen, wie Wissen aus DIP im Gesundheitswesen besser extrahiert und kommuniziert werden kann. Dazu wird ein bestehender Ansatz zur Systematisierung gestaltungsorientierter Forschungsprojekte, das Design Science Grid, in drei Fallstudien angewendet. Aus dieser Anwendung werden sieben

\footnotetext{
T. Scheplitz $(\bowtie) \cdot$ M. Benedict $\cdot$ H. Schlieter $\cdot$ S. Kaczmarek $\cdot$ M. Susky Lehrstuhl für Wirtschaftsinformatik, insbes. Systementwicklung, Fakultät für Wirtschaftswissenschaften, Technische Universität Dresden, 01062 Dresden, Deutschland E-Mail: tim.scheplitz@tu-dresden.de

M. Benedict

E-Mail: martin.benedict@tu-dresden.de

H. Schlieter

E-Mail: hannes.schlieter@tu-dresden.de

S. Kaczmarek

E-Mail: stefanie.kaczmarek@tu-dresden.de

M. Susky

E-Mail: marcel.susky@tu-dresden.de
} 
verschiedene Typen von Wissen beschrieben, die aus DIP im Gesundheitswesen resultieren können. Für künftige Praxis-Forschungs-Projekte wird damit ein Weg zur Systematisierung von Ergebnissen aufgezeigt. Die gefundenen Wissenstypen können dabei als Ausgangspunkt einer Klassifizierung von zu erzielenden Kontributionen dienen.

Schlüsselwörter Digitale Innovationsprojekte $\cdot$ Multi-Fallstudie $\cdot$ Wissenstypen · Design Science Research

\title{
Research in Digital Innovation Projects-Between Practicability and Scientific Relevance
}

\begin{abstract}
Project results of digital innovation projects (DIP) develop innovative artefacts of different shapes and forms which are strongly reminiscent of results from design-oriented research activities (design science). Nevertheless, the findings from DIP in the healthcare sector often do not find their way into the scientific debate. Although the implementation of such projects is strongly promoted in the health care system, design recommendations or even standards for communicating the results have not been established. Explicit and implicit knowledge, as project results in addition to the innovation itself, can be of critical importance for the organization and implementation of similar projects. Their communication and structured provision have so far been little addressed in science. The aim of this paper is therefore to show a way how knowledge from DIP can be better extracted and communicated. For this purpose, an existing approach to systematize design-oriented research projects, the Design Science Grid, will be applied in three case studies. Seven different types of knowledge that can result from DIP in health care are described. For future practice-oriented research projects, a way to systematize results is thus shown. The knowledge types found can serve as a starting point for a classification of project goals.
\end{abstract}

Keywords Digital Innovation Projects - Multi-Case Study · Knowledge Types · Design Science Research

\section{Einleitung}

Im Zeitalter der Digitalen Transformation des öffentlichen, privaten und wirtschaftlichen Lebens erfährt der wirtschaftsinformatorischen Forschung eine wichtige Bedeutung diesen Wandel zu erklären und zu gestalten. Dennoch bestimmt auch die Debatte zur Praxisrelevanz von Forschungsergebnissen und die wissenschaftliche Qualität von innovativen Praxisarbeiten den aktuellen Diskurs. Digitale Innovationsprojekte (im weiteren Verlauf als „DIP“ abgekürzt) im Gesundheitswesen gelten innerhalb dieses Wandels als wesentliche Triebkraft (Laurenza et al. 2018). Ihre Projektergebnisse sind typischerweise Kombinationen aus integrierten Versorgungsmodellen und innovativen Systemlösungen (Digitale Artefakte) unterschiedlicher Form und Ausprägung. Diese Ergebnisse ähneln in ihrer Art den Resultaten ge- 
staltungsorientierter Forschungstätigkeit (in der Fachwelt auch als Design Science Research tituliert) (Österle et al. 2011; Hevner et al. 2019).

Die Untersuchung von Werner (2019) unterstreicht eine Diskrepanz, die zwischen den zur Umsetzung verwendeten Forschungsmethoden und solchen, die international als publikationswürdig anerkannt sind, besteht. Während zu den tatsächlich verwendeten Forschungsmethoden Systementwicklungsansätze wie das Prototyping, Umfragen bzw. Interviews gehören, zählen zu den häufig publizierten Forschungsmethoden die konzeptuelle Deduktion als auch die quantitativ/qualitativ-empirische Forschung (Werner 2019).

Gleichzeitig lässt sich beobachten, dass sich die Relevanz und Bedeutung von rein wissenschaftlich entwickelten Methoden für die Forschungsergebnisse marginalisieren. Nicht umsonst gilt die Evaluation von Design Science-Kontributionen abseits von konstruierten Szenarien, als zentrale Herausforderungen der Wissenschaftsgemeinschaft (Frank 1998; Venable et al. 2012). Beispielsweise werden in DIP im Gesundheitswesen häufig genannte Forschungsmethoden (Prototyping und qualitativ-empirische Forschung) angewendet (Fichman et al. 2014). Die mangelnde Anerkennung solcher Forschungsergebnisse führt jedoch nur zu einem schwachen Transfer bzw. Verschränkung praxisorientierter Forschung und akademischer Verwertung. Als Resultat erhalten Erkenntnisse aus DIP im Gesundheitswesen nur schwerlich Einzug in die Wissenschaft. Hingegen ist gerade die Auseinandersetzung mit Defiziten, welche die Umsetzung von DIP im Gesundheitswesen betreffen, für die Forschung und Praxis unerlässlich bspw. zur Untersuchung neuer Versorgungsmodelle und Erforschung theoretischer Erklärungsansätze.

Innerhalb von DIP entsteht neben explizitem Wissen häufig auch implizites Wissen, welches durch Externalisierung (Otto and Österle 2010) für die Organisation und Durchführung von Nachfolgeprojekten bedeutend sein kann. Insbesondere spielen der Umgang mit implizitem sowie explizitem Wissen und dessen Aufbereitung eine wichtige Rolle für die Reichweite und letztlich auch für die Implikationen die aus Forschungs- und Praxisprojekten erwachsen. Der vorliegende Beitrag widmet sich diesen Themen und stellt die Fragen

1. Wie kann Wissen aus praxisorientierten Konsortialprojekten im Gesundheitswesen systematisch erfasst und explizieren werden?

2. Wie kann das gewonnene Wissen für zukünftige DIP im Gesundheitswesen aufbereiten werden?

Ziel des Beitrags ist die Stärkung der Präsenz von DIP innerhalb der wissenschaftlichen Diskussion und Verbesserung der Akzeptanz ihrer Erkenntnisse innerhalb wissenschaftlicher Publikationen. Hierzu wird im vorliegenden Beitrag der aktuelle wissenschaftliche Diskurs zusammengefasst und eine Typologie von Wissensbeiträgen von gestaltungsorientierten Projekten vorgestellt. Mit ihrer Hilfe sind eine systematische Klassifizierung und Beschreibung von DIP sowie der dazugehörigen Wissensbeiträge möglich. Anhand von drei Fallbeispielen wird zudem gezeigt, wie diese Systematisierung auch praktisch genutzt werden kann. Darüber hinaus werden auf Basis der Fallbeispiele Archetypen für den Wissensbeitrag innerhalb Digitaler Gesundheitsinnovationen skizziert. 
Dieser Beitrag gliedert sich wie folgt: Der Einleitung folgen die Grundlagen zu Digitalen Innovationen und deren Verknüpfung mit dem Gesundheitswesen in Abschn. 2. In Abschn. 3 wird der aktuelle Stand der Forschung zu Wissenstypen in der Wirtschaftsinformatikforschung zusammengefasst und das ,Design Science Research Grid“" als Systematisierungsansatz für DIP vorgestellt. Anschließend werden im Rahmen einer Fallstudie, drei Projekte aus der Integrierten Versorgung beschrieben. Es folgt eine exemplarische Systematisierung von Wissensbeiträgen und die Ableitung von potentiellen Wissenstypen (Abschn. 4). Der Beitrag endet mit einer kritischen Diskussion und gibt einem Ausblick auf künftige Forschungsvorhaben.

\section{Digitale Innovationen im Gesundheitswesen}

Bei einer Digitalen Innovation handelt es sich um eine Produkt-, Service- oder Geschäftsmodellinnovation, die an den Einsatz von digitaler Technologie geknüpft ist. Eine Digitale Innovation kann einerseits als Mittel im Innovationsentwicklungsprozess betrachtet werden und andererseits als Ergebnis. Das Phänomen der Digitalen Innovation umfasst neue digitale Technologien, die Digitalisierung von Informationen, die digital ermöglichte Generativität und ein Innovationsmanagement mit einer größeren Reichweite von Innovationen über Unternehmensgrenzen hinweg (Yoo et al. 2010).

Digitale Innovationen haben in den vergangenen Jahren verstärkt Einzug in vielen Bereichen des individuellen und gesellschaftlichen Lebens erhalten und stellen eine treibende Kraft für Transformationsprozesse dar. Während Digitale Innovationen sich in anderen Branchen früh verbreiteten und sich weiterhin rasch entwickeln, bleibt der Einsatz von digitalen Technologien im Gesundheitssektor nach wie vor hinter seinen Möglichkeiten zurück. Ein Grund dafür ist in der Schwierigkeit zu sehen, ein solch komplexes, historisch gewachsenes und hoch reguliertes System wie das Gesundheitssystem durch digitale Technologien mit kurzen Produktlebenszyklen abrupt, spürbar und nachhaltig zu verbessern. Trotz der komplexen Rahmenbedingungen bleibt der Handlungsbedarf bestehen.

In Anbetracht der alternden Gesellschaft, der Zunahme multimorbider Krankheitssituationen sowie dem anhaltenden Fachkräftemangel sind innovative Ideen zur Neuausrichtung des Gesundheitssektors gefragt. Mit der Verabschiedung des Gesetzes zur Stärkung der Versorgung in der gesetzlichen Krankenversicherung hat die Bundesregierung einen wesentlichen Schritt dazu beigetragen. Es setzt u. a. stärkere Anreize für die Integration von Digitalen Innovationen in der Versorgung sowie in der Versorgungsforschung und adressiert insbesondere unterversorgte oder strukturschwache Gebiete. Das kürzlich verabschiedete Digitale Versorgungsgesetz schließt sich dieser Bewegung an und fördert u. a. die Einführung Digitaler Innovationen in den Gesundheitsmarkt.

Die Gesetzgebung hat grundsätzlich den Handlungsbedarf ihrerseits erkannt, die Freisetzung der vielseitigen Potenziale Digitaler Innovationen zu beschleunigen. Neben der Erprobung bzw. Etablierung von neuen zeitgemäßen und bedarfsgerechten intersektoralen Versorgungsmodellen ermöglichen sie auch die Verbesserung der Versorgungsqualität (Herrmann et al. 2018). Sie können über den gesamten 
Leistungserbringungsprozess hinweg integriert werden. Im Bereich der Prävention fördern digitale Anwendungen bspw. mehr Bewegung oder eine gesündere Ernährung. Im Rahmen der Diagnostik können sie der Entscheidungsunterstützung der Ärzte dienen oder auf Ebene der Therapie mit Hilfe von Medikationsplänen Unterstützung leisten. Ebenfalls sind Digitale Innovationen im Zuge der Rehabilitation, indem Virtual Coaches eingesetzt werden, denkbar. Um die benannten Potenziale auszuschöpfen, bedarf es wiederum einer stärkeren Verzahnung der einzelnen Versorgungssektoren. Derzeit agieren die einzelnen Sektoren und Fachdisziplinen zumeist unabhängig voneinander und die Patientenzentriertheit steht nicht im Vordergrund ihrer Aktivitäten. Dabei bedarf insbesondere die Versorgung multimorbider und chronischer Krankheitsbilder eine unterbrechungsfreie und aufeinander abgestimmte Behandlung, da bei Mehrfacherkrankungen häufiger Komplikationen auftreten als bei Routineprozeduren (Matusiewicz et al. 2017).

\section{Gestaltungswissen in Digitalen Innovationsprojekten}

\subsection{Grundlagen des Gestaltungswissens}

Forschungsseitig lässt sich die Wirtschaftsinformatik über zwei verschiedene Ziele erklären: dem Erkenntnisziel und dem Gestaltungsziel (Becker et al. 2003). Beide Ziele fokussieren auf die Generierung von Wissen. Dabei ist die Form des resultierenden Wissens jedoch für beide Ziele unterschiedlich. Das erste Ziel fokussiert die Erzeugung beschreibenden Wissens (deskriptives Wissen). Deskriptives Wissen trägt dazu bei, Phänomene unterschiedlicher Natur durch Beobachtung, Klassifikation, Messung oder Katalogisierung zu begreifen. Infolgedessen lassen sich daraus Erkenntnisse in Form von Naturgesetzen, Prinzipien und Theorien ableiten. Ein Gestaltungsziel adressiert die Entwicklung von Gestaltungswissen (präskriptives Wissen). Im Gegensatz zum deskriptiven Wissen bezieht sich das präskriptive Wissen darauf, wie bestimmte Probleme gelöst (z. B. technische Regelwerke) oder bestimmte soziotechnische Systeme entwickelt werden können (z. B. Systemanforderungen, Architekturkonzepte). Das präskriptive Wissen kann seinerseits in Lösungsdesignentitäten und Lösungsdesignwissen unterteilt werden. Unter Lösungsdesignentitäten versteht man Artefakte wie Modelle und Methoden, aber auch Artefaktinstanzen, Designprozesse und Artefaktentwicklungsprozesse. Lösungsdesignwissen kann in Form von technologischen Regeln, Wissen zur Realisierung von Entitäten (Anforderungen, Prinzipien, Merkmale) sowie Wissen für Designprozesse (Methoden, Techniken) festgehalten werden (Drechsler and Hevner 2018).

Beschreibendes und gestaltendendes Wissen stehen in einem engen Zusammenhang. Deskriptives Wissen unterstützt beispielsweise bei der Problemdefinition als auch bei der Umsetzung von Gestaltungszielen. So können identifizierte Verhaltensweisen von Nutzern (deskriptives Wissen) bei der Beschreibung von Gestaltungsregeln für Mensch-System-Interaktion berücksichtigt werden (Drechsler and Hevner 2018).

In Abgrenzung zu dem Wissen aus der allgemeinen Wissensbasis der Wirtschaftsinformatik steht das Wissen, welches aus einzelnen Projekten stammt. DIP im Ge- 
sundheitswesen bilden dabei einen konkreten Typ von Projekten, die als spezifischen Problemraum den Einsatz digitaler Lösungen im Gesundheitswesen adressieren. Wissen aus solchen Projekten wird im folgenden Projektwissen genannt und weist ein eher zeitweiliges, nicht bzw. nur im Projektkontext erprobtes hochspezifisches Wesen auf. Dieses Wissen umfasst die Projektergebnisse als auch die Erfahrungen und impliziten Erkenntnisse der einzelnen Stakeholder.

Wissen aus Innovationsprojekten kann sowohl in deskriptives als auch in präskriptives Wissen transformiert werden. Diese Transformation erfolgt durch die systematische Gestaltung von Artefakten und der darauf aufbauenden Evaluation von Gestaltungsergebnissen (March and Smith 1995). Darüber hinaus gibt es weitere Wissensquellen, aus denen das Wissen für DIP gewonnen werden kann, z. B. aus dem eigenen Erfahrungsschatz oder aus der Zusammenarbeit in Konsortien und kreativen Tätigkeiten. Der Bereich des Projektwissens enthält Wissen über den Problemund Lösungsraum eines Projekts. Kenntnisse über den Problemraum umfassen das Wissen um Zusammenhänge, die Problemidentifikation und Gütekriterien. Die Entwicklung solchen Wissens ist dabei nicht immer direkt durch methodische Planung generierbar, sondern ergibt sich auch aus dem nicht vollständig vorhersehbaren Projektverlauf. Beispielsweise kann eine für ein konkretes Problem geschaffene Softwarearchitektur durch eine nachträgliche Generalisierung für eine Problemklasse aufbereitet und als wiederverwendbares Konstrukt der Wissensbasis zurückgeführt werden. Hierbei muss das Generalisierungs-potential erkannt, das entstandene Projektwissen expliziert und transformiert werden. Dabei ist die Generalisierung sowohl des Problems als auch der Lösung notwendig (Drechsler and Hevner 2018).

Bis Forschende ein genaues Problemverständnis entwickelt und den Problemkontext durchdrungen haben, werden im Projektkontext mehrere Iterationen der Wissensanhäufung durchlaufen. So nutzen, produzieren (durch Ersetzen/ Weiterentwickeln) und verwerfen sowohl Praktiker als auch Wissenschaftler diverse Wissenseinheiten ehe eine endgültig passfähige Lösung gefunden ist. Dieser Sachverhalt dient als Motivation bzw. Anknüpfungspunkt, sich im Rahmen der vorliegenden Arbeit mit dem Bedarf der Transformation von Wissen in eine konkrete Domäne zu beschäftigen (Otto and Österle 2010). Die Anwendung von Wissen aus der Wissensbasis in die Versorgungsrealität und vice versa (Wissenstransformation) kann auf verschiedenen Wegen erfolgen und unterschiedlich dokumentiert sein (siehe Abb. 1). Beispielsweise können Systemspezifikationen (oder einzelne Bestandteile) oder auch immaterielle Ideen einzelner Forscher Gegenstand dieses Transformationsprozesses sein. Bei diesem Prozess wird jedoch nicht nur auf etabliertes Wissen zurückgegriffen, sondern auch auf ungetestetes und temporäres Wissen, welches im Rahmen von unstrukturierten, kreativen und heuristischen Verfahrensweisen erzeugt wurde. Antizipiert wird dabei, dass dieses Wissen im Zuge der Projektzusammenarbeit geschaffen und vereinzelt unter Mitgliedern geteilt wurde. Gegenüber der Außenwelt werden typischerweise die Projektendergebnisse offengelegt, nicht jedoch die Überlegungen und Erfahrungen, die bei der Erzeugung dieser eine Rolle spielen (Drechsler and Hevner 2018).

Es ist möglich, die Wissenstransformation aus DIP näher zu formalisieren. Hierfür kommen vier verschiedene Formen der Wissenstransformation in Betracht: die direkte Übernahme von Wissen (1), die Abstraktion (2), die Generalisierung (3) und 


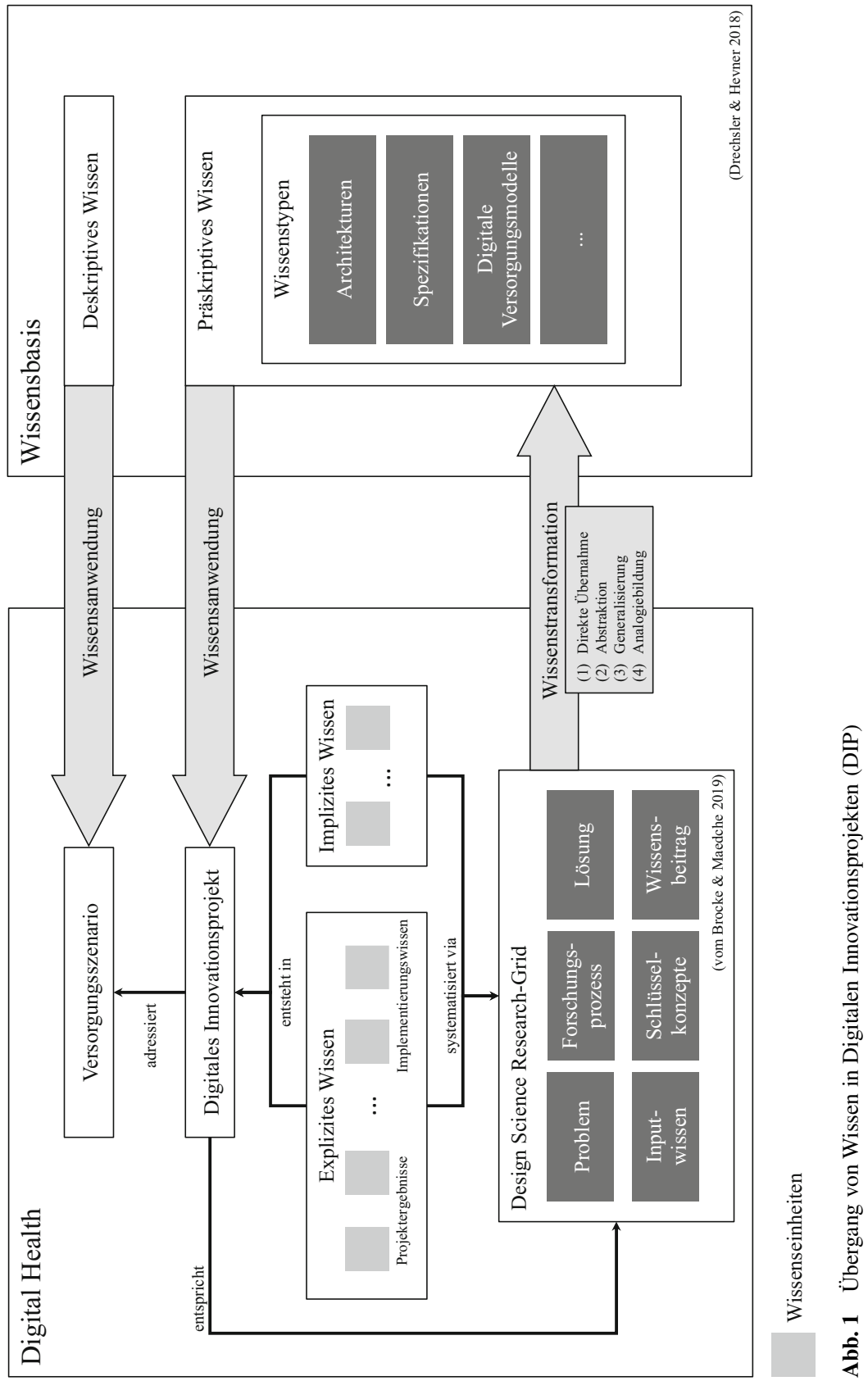


die Analogiebildung (4). Bei der direkten Übernahme des Wissens erfolgt die Einszu-Eins-Wiedergabe der im Rahmen eines DIP gewonnenen Erkenntnisse. Bei der Abstraktion dagegen werden anhand gewonnener Erkenntnisse aus DIP allgemeingültige Regeln und Konzepte abgeleitet, z. B. in Form einer Referenzarchitektur aus konkreten heterogenen Architekturen. Unter Generalisierung ist die Verallgemeinerung gleichartiger Wissenseinheiten aus spezifischem Wissen zu verstehen, z. B. die Ableitung einer domänenspezifischen Referenzarchitektur. Bei der Analogiebildung wird das neuerschaffene Wissen derart aufgearbeitet, dass es in Bezug auf ein spezifisches Merkmal im Vergleich zum bestehenden Wissen als ähnlich wahrgenommen wird.

\subsection{Design Science Research-Grid}

DIP sind durch eine rasante Weiterentwicklung der neusten Informations- und Kommunikationstechnologien und variierende Anforderungen gekennzeichnet. Eine sorgfältige und angemessene Planung der praktischen Projektverläufe ist folglich eine beinah selbstverständliche Kernaufgabe des Projektmanagements. Bei kombinierten Forschungs-Praxis-Projekten sollten auch intendierte Forschungsergebnisse sinnvoll strukturiert und geplant werden. Vom Brocke and Maedche (2019) schlagen in diesem Zusammenhang das Design Science Research Grid vor, das aus sechs Kerndimensionen besteht (Tab. 1). Es soll Forschende bei der effektiven Planung und Kommunikation des Design Science Research-Projekts unterstützen, indem die wichtigsten Aspekte strukturiert beschrieben und abgebildet werden. $\mathrm{Zu}$ den sechs Kerndimensionen, die projektspezifisch gewichtet und angeordneten werden können, gehören die Problem- und Lösungsbeschreibung, das entsprechende Input- und Outputwissen sowie der Forschungsprozess inkl. Schlüsselkonzepten.

Die Problembeschreibung dient der anschließenden Identifikation möglicher Lösungen für ein konkretes Problem, welches sich in einem Problemraum mit entsprechendem Kontext (Domäne, Stakeholder, Zeit und Ort, Gütekriterien) bewegt. Das Input- bzw. Outputwissen (weiter als Wissensbeitrag bezeichnet) bezieht sich auf das genutzte Vorwissen sowie dasjenige Wissen, welches Ergebnis eines Design Science Research Projekts bzw. eines DIP ist. Wie im vorherigen Kapitel beschrieben, kommen hierfür verschiedene Wissensbasen in Frage, z. B. deskriptives oder präskriptives Wissen. Der Forschungsprozess adressiert die zur Lösung des konkreten Problems vorgesehenen und notwendigen Forschungsaktivitäten. Unterscheiden

Tab. 1 Design Science Grid nach vom Brocke and Maedche (2019)

\begin{tabular}{|c|c|c|}
\hline \multicolumn{3}{|c|}{ Name des gestaltungsorientierten Projekts } \\
\hline $\begin{array}{l}\text { Problem: } \\
\text { Konkrete Problembeschreibung, } \\
\text { welche das Problem konkret } \\
\text { innerhalb der Domäne verortet }\end{array}$ & $\begin{array}{l}\text { Forschungsprozess: } \\
\text { Expliziertes Vorgehen } \\
\text { der gestaltungsorientier- } \\
\text { ten Forschung }\end{array}$ & $\begin{array}{l}\text { Lösung: } \\
\text { Resultat des Erkenntnisprozesses } \\
\text { im Sinne von Gestaltungsprinzipien, } \\
\text { generischen Lösungsartefakten }\end{array}$ \\
\hline $\begin{array}{l}\text { Inputwissen: } \\
\text { Zugrundeliegendes Inputwissen } \\
\text { im Sinne von beschreibenden } \\
\text { Theorien und präskriptiven Ge- } \\
\text { staltungswissens }\end{array}$ & $\begin{array}{l}\text { Schlüsselkonzepte: } \\
\text { Schlüsselkonzepte, die } \\
\text { zur Umsetzung des Pro- } \\
\text { jektes verwendet werden }\end{array}$ & $\begin{array}{l}\text { Wissensbeitrag: } \\
\text { Gestaltungswissen, welches sich } \\
\text { innerhalb des Forschungsprojekt } \\
\text { generiert und zur Problemlösung } \\
\text { beigetragen hat }\end{array}$ \\
\hline
\end{tabular}


lassen sich hier bspw. Entwicklungs- und Evaluationsaktivitäten sowie Forschungsmethoden, wie Literaturrecherchen. Wesentliche Konzepte, auf die im Verlauf des Design Science Research Projekts bzw. DIP zurückgegriffen wird, bilden die Schlüsselkonzepte. Die Lösungsbeschreibung adressiert die Lösung des Problems, indem es die Form der Lösung (Konstrukt, Model, Methode, Instanz) näher spezifiziert (vom Brocke and Maedche 2019).

\subsection{Fallbetrachtung Digitaler Innovationsprojekte im Gesundheitswesen}

Die nachfolgend beschriebenen Projekte sind den Bereichen der Integrierten Versorgung und Telemedizin zuzuordnen. Unter den Projektpartnern befinden sich Experten aus den Fachgebieten Medizin sowie der System- und Softwareentwicklung. Darüber hinaus erfolgt eine Kooperation mit den zentralen Informations- und Projektmanagementeinrichtungen der Krankenhäuser. Einige Projektpartner sind in mehreren Projekten involviert, z.B. ein Anbieter von medizinischen Fallunterlagen und das örtliche Universitätsklinikum. Im Rahmen der Multi-Fallstudie werden drei Projekte betrachtet, die als erklärende Anwendungsfälle der Untersuchung dienen. Alle drei Projekte haben die Entwicklung mindestens einer Digitalen Innovation im Gesundheitswesen für eine Integrierte Versorgungsumgebung, ihre Verbreitung in die Gesundheitspraxis und damit ihre Integration in die bestehende Informationssystemlandschaft zum Ziel.

Ein gemeinsames Ziel der Projekte ist die Etablierung einer krankheitsspezifischen elektronischen Fallakte. Nebst internen Strukturen unterscheiden sich die Projekte in der Art und Weise, wie sie von Informationssystemen betroffen sind. Außerdem werden verschiedene digitale Lösungen in diesem Zusammenhang realisiert. In den Projekten werden unterschiedliche Krankheitsbilder betrachtet. Dabei erfordert jedes Krankheitsbild für sich einen anderen Lösungsansatz und insbesondere auch andere IT-Artefakte. Im Folgenden werden die wesentlichen Inhalte der drei Projekte vorgestellt und in Tab. 2 zusammengefasst.

Das Projekt STROKE zielt auf die informationelle Verbindung zwischen einer existenten eHealth-Plattform mit schlaganfallspezifischen Diensten und IT-Systemen von Hausärzten sowie Spezialisten für die ambulante Nachsorge. Hierdurch soll der Nachsorgeprozess von Schlaganfallpatienten verbessert werden, indem ein integrierter Informationsfluss zwischen Case Managern und Hausärzten ermöglicht wird. Im Mittelpunkt dieses Kommunikationsszenarios steht ein schlaganfallspezifisches Clinical Document Architecture-Dokument (CDA Schlaganfallpass), das von allen beteiligten Leistungserbringern der Nachsorge gemeinsam genutzt wird.

Das Projekt NEURO beschäftigt sich mit der Entwicklung eines Integrierten Versorgungsportals für Patienten der Multiplen Sklerose, einer chronischen neurologisch degenerativen Erkrankung. Das Hauptziel ist es, eine bessere Verbindung zwischen Fachkräften, Patienten sowie (informell) unterstützenden Pflegediensten herzustellen. So werden Patienten und Angehörige bei der Krankheitsbewältigung besser unterstützt. Portalnutzer sollen Zugang zu ihren Fallakten erhalten und individuelle, kontextsensitive Dienste nutzen können, z. B. Erinnerungsfunktionen für Medikamente, therapeutische Übungen und spezifische Fragebögen. 


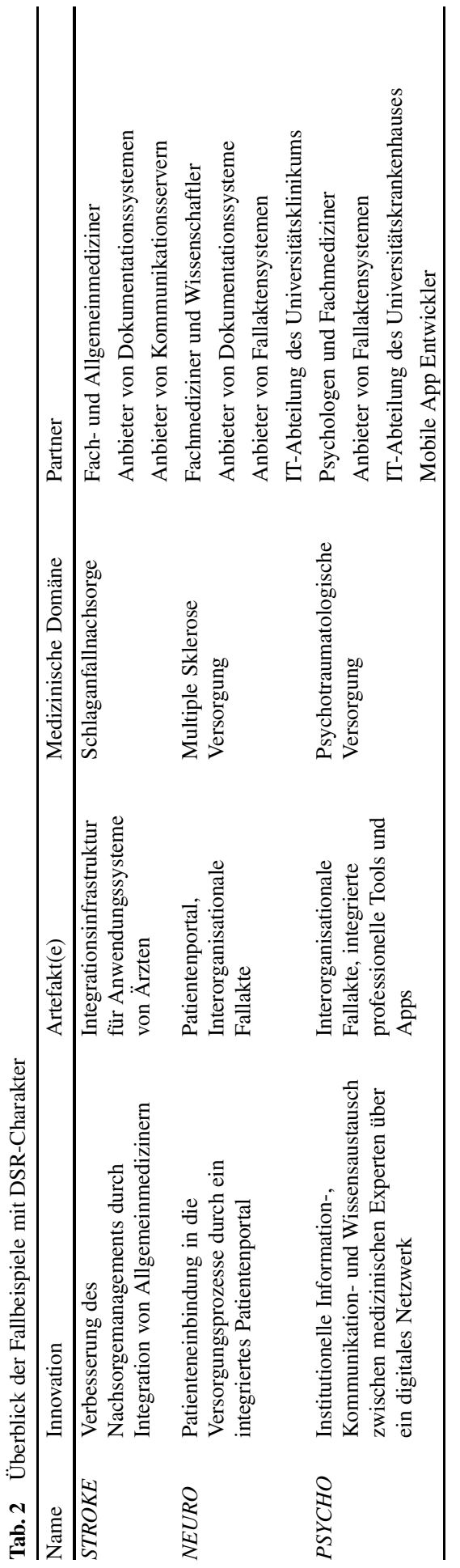


Das Projekt $P S Y C H O$ zielt auf eine Verbesserung der interorganisationalen Behandlung von psychotraumatologischen Patienten ab. Mit Hilfe moderner Informations- und Kommunikationstechnologien wird die Kommunikation aller Beteiligten über relativ große Entfernungen hinweg optimiert. Infolgedessen soll eine fallspezifische Dokumentation institutsübergreifend verfügbar gemacht werden. Zusätzliche Instrumente und Verfahren für ein standardisiertes Screening und eine standardisierte Diagnose werden entwickelt und bewertet.

\subsection{Methodik zur Systematisierung der Wissensbeiträge}

Zwischen dem von Drechsler and Hevner (2018) vorgeschlagenen konzeputellen Framework, dass die Nutzung, die Produktion bzw. den Beitrag von Wissen veranschaulicht (Abschn. 3.1), und dem Design Science Research Grid (Abschn. 3.2) nach vom Brocke and Maedche (2019) bestehen deutliche Parallelen. Am Anfang eines Innovationsprojekts steht stets die Beschreibung des Problemraums. Der Problemraum enthält in beiden Konzepten Aussagen über den Projektkontext, die konkreten Probleme und Gütekriterien. Gemäß vom Brocke and Maedche (2019) kann Inputwissen sowohl deskriptives als auch präskriptives Wissen sein. Bei Outputwissen handelt es sich um rein gestaltungsorientiertes Wissen. Dieses gestaltungsorientierte Wissen bildet die Grundlage für die Lösungsraumbeschreibung. Drechsler eand Hevner (2018) dagegen sehen Inputwissen als rein deskriptives Wissen an. Die im

Tab. 3 Design Science Research Grid für das Projekt STROKE

\begin{tabular}{|c|c|c|}
\hline \multicolumn{3}{|l|}{ STROKE } \\
\hline $\begin{array}{l}\text { Problem: } \\
\text { - Konkrete Probleme: Fehlen- } \\
\text { de Integration von Haus- und } \\
\text { Fachärzten in eine bestehende } \\
\text { digitale Telemedizinplattform } \\
\text { für die ambulante Nachsorge } \\
\text { - Domäne/Krankheitsbild: Schlag- } \\
\text { anfall } \\
\text { - Stakeholder: Haus- und Fachärz- } \\
\text { te sowie weiterbehandelnde } \\
\text { Fachgruppen }\end{array}$ & $\begin{array}{l}\text { Forschungsprozess: } \\
\text { - Analyse und Entwick- } \\
\text { lung von Versorgungs- } \\
\text { prozessen und Patien- } \\
\text { tenpfaden } \\
\text { - Systementwicklung der } \\
\text { Integrationsarchitektur } \\
\text { zur Integration von } \\
\text { Hausärzten } \\
\text { - Evaluation der Inte- } \\
\text { grationslösung mit } \\
\text { Pilotpraxen }\end{array}$ & $\begin{array}{l}\text { Lösung: } \\
\text { - Erweitertes klinische Pfad- } \\
\text { modell für die Integration } \\
\text { hausärztlicher Leistungen in } \\
\text { die Schlaganfallnachsorge } \\
\text { - Systemarchitektur zur Integra- } \\
\text { tion der beteiligten Arztpraxis- } \\
\text { systeme } \\
\text { - Integrationsmodelle für ver- } \\
\text { schiedene Typen von Arztpra- } \\
\text { xen } \\
\text { - Erweiterter digitaler Schlagan- } \\
\text { fallpass }\end{array}$ \\
\hline $\begin{array}{l}\text { Inputwissen: } \\
\text { - Medizinisches Fachwissen über } \\
\text { Erkrankung und ihre Behand- } \\
\text { lung } \\
\text { - Standardisierte Infrastruktur } \\
\text { (Telemedizinplattform) für die } \\
\text { Schlaganfall-Akutversorgung } \\
\text { - Integrierter Versorgungspfad: } \\
\text { Akutphase bis ambulante Nach- } \\
\text { sorge } \\
\text { - Schnittstellenformate und Tech- } \\
\text { nische Standards (IHE XDS.b; } \\
\text { HL7 CDA) }\end{array}$ & $\begin{array}{l}\text { Schlüsselkonzepte: } \\
\text { - Informationsobjekte } \\
\text { und -flüsse } \\
\text { - Organisatorische und } \\
\text { technische Einbindung } \\
\text { von Leistungserbrin- } \\
\text { gern } \\
\text { - Anwendungssysteme } \\
\text { - Schnittstellenspezifikationen- } \\
\text { - }\end{array}$ & $\begin{array}{l}\text { Wissensbeitrag: } \\
\text { - WS1. Technische Spezifikation } \\
\text { der Systemintegration } \\
\text { - WS2. Architekturmodelle inkl. } \\
\text { Schnittstellenkonzept } \\
\text { - WS3. Vorgehensmodell zur } \\
\text { Einbindung niedergelassener } \\
\text { Ärzte } \\
\text { - WS4. Stufenkonzept zur In- } \\
\text { tegration von Hausärzten in } \\
\text { einrichtungsübergreifende } \\
\text { Versorgungskonzepte }\end{array}$ \\
\hline
\end{tabular}


Tab. 4 Design Science Research Grid für das Projekt NEURO

\begin{tabular}{|c|c|c|}
\hline EURO & & \\
\hline $\begin{array}{l}\text { Problembeschreibung: } \\
\text { - Konkrete Probleme: Bedarf } \\
\text { an ortsungebundenen Ver- } \\
\text { sorgungsprozessen; Defizite } \\
\text { in der Einbindung formeller } \\
\text { und informeller Teilhaber } \\
\text { - Domäne/Krankheitsbild: } \\
\text { Multiple Sklerose } \\
\text { - Stakeholder: Patienten, } \\
\text { informelle und formelle } \\
\text { Leistungserbringer }\end{array}$ & $\begin{array}{l}\text { Forschungsprozess: } \\
\text { - Systematische Prozessana- } \\
\text { lyse des interorganisationa- } \\
\text { len Patientenpfads } \\
\text { - Durchführung Patienten- } \\
\text { befragung } \\
\text { - Ableitung Gestaltungs- } \\
\text { empfehlungen } \\
\text { - Agiles Prototyping eines } \\
\text { pfadbasierten Patientenpor- } \\
\text { tals } \\
\text { - Entwicklung wiederver- } \\
\text { wendbarer Referenzmodelle } \\
\text { und Entwurfsmuster }\end{array}$ & $\begin{array}{l}\text { Lösung: } \\
\text { - Patientenportal } \\
\text { - Implementierung von Patientenpfa- } \\
\text { den in ein Patientenportal } \\
\text { - Systemarchitektur zur Integration } \\
\text { verschiedener Anwendungssysteme } \\
\text { - Spezifikation einer MS-Fallak- } \\
\text { tenstruktur, Austauschformate und } \\
\text { Schnittstellen }\end{array}$ \\
\hline $\begin{array}{l}\text { Inputwissen: } \\
\text { - Medizinisches Fachwissen } \\
\text { über Erkrankung und ihre } \\
\text { Behandlung } \\
\text { - Methodenwissen Agile } \\
\text { Softwareentwicklung } \\
\text { - Multiple Sklerose Doku- } \\
\text { mentationssystem } \\
\text { - Patient Empowerment } \\
\text { - IT-Standards der Medizin- } \\
\text { informatik } \\
\text { - Elektronische Fallakte } \\
\text { - Application Programming } \\
\text { Interface Design-Ansätze }\end{array}$ & $\begin{array}{l}\text { Schlüsselkonzepte: } \\
\text { - Patientenpfade } \\
\text { - Softwarearchitektur } \\
\text { - Schnittstellenspezifikation }\end{array}$ & $\begin{array}{l}\text { Wissensbeitrag: } \\
\text { - WN1. Generische Portalkomponen- } \\
\text { ten } \\
\text { - WN2. Abbildung von Patientenpfa- } \\
\text { de via FHIR } \\
\text { - WN3. Bedarfe von MS-Patienten } \\
\text { hinsichtlich der digitalen Integration } \\
\text { in den Behandlungsverlauf } \\
\text { - WN4. Referenzarchitektur für die } \\
\text { Patientenintegration } \\
\text { - WN5. Wiederverwendbare Digital } \\
\text { Health-Muster zur Patientenintegrati- } \\
\text { on } \\
\text { - WN6. Usability-Empfehlungen } \\
\text { - WN7. Scrum im Digital Health } \\
\text { Kontext }\end{array}$ \\
\hline
\end{tabular}

Rahmen von DIP verwendeten Forschungsprozesse und Schlüsselkonzepte sind bezogen auf das konzeptuelle Framework als ein Teil des Lösungsraums zu verstehen.

Anhand der vorab beschriebenen Fallbetrachtungen (Abschn. 3.3) ist das in den einzelnen DIP gesammelte Wissen mittels des Design Science Research-Grids systematisiert (siehe Tab. 3, 4 und 5). Dabei sind insbesondere die Wissensbeiträge je DIP hervorgehoben (grau hinterlegt), aus welchen die grundlegenden Wissenstypen im nachfolgenden Kapitel resultieren. Ihre Identifikation erfolgt induktiv aus den einzelnen in den Projekten identifizierten Wissensbeiträgen (siehe Abschn. 3.5). Die einzelnen Inhalte in den projektspezifischen Design Science Research-Grids wurden durch eine Analyse der Ergebnisdokumente, Protokolle und anderer Projektunterlagen des jeweiligen Projektes ermittelt. Soweit entstandenes Wissen innerhalb der Projektdokumentation expliziert wurde, konnte dieses in das entsprechende Grid direkt übertragen werden. Implizites Wissen kann jedoch selten alleinig über den Ansatz der Projektarchäologie identifiziert werden. Daher wurden in Diskussionen innerhalb der Projektkonsortien bzw. innerhalb abgrenzbarer Gruppen die Projekte, Verläufe, Ergebnisse und bereits expliziertes Wissen reflektiert. Die theoretischen Hintergründe des Gestaltungswissens (Abschn. 3.1) sowie die innerhalb des Grids involvierten Aspekte inklusive ihrer Systematisierung (Abschn. 3.2) dienten hierbei 
Tab. 5 Design Science Research Grid für das Projekt PSYCHO

\begin{tabular}{lll}
\hline PSYCHO & & \\
\hline Problembeschreibung: & Forschungsprozess: & Lösung: \\
- Konkrete Probleme: & - Fokusgruppen zur Konzep- & - Spezifikation der Fallakten- \\
Verbesserungswürdi- & tion einer elektronischen & struktur, Austauschformate und \\
ge Kommunikation und & Fallakten & Schnittstellen \\
Interoperabilität in der & - Prototypenerstellung & - Anforderungsprofil für eine elek- \\
Behandlungskette von & - Entwicklung von Integra- & tronische Fallakte in der Psy- \\
Traumafolgestörungen & tionskonzepten für Partner & chotraumatologie \\
- Domäne/Krankheitsbild: & eines interorganisationalen & - Systemarchitektur zur Integration \\
Psychotraumatologie & Netzwerks & von Patienten- und Diagnose-Apps \\
- Stakeholder: professio- & - Requirements Engineering & - FHIR-Fragebögen für die psy- \\
nelle Versorgungsteilhaber & für einrichtungsübergreifen- & chotraumatologische Aufnahme \\
& den Fallakte & \\
& - Spezifikation der Austauch- & \\
& und elektronischen Doku- & \\
& mentenformate & \\
Inputwissen: & Schlüsselkonzepte: & Wissensbeitrag: \\
- Wissen über die Erkran- & - Interorganisationales Infor- & - WP1. Psychotraumatologische \\
kung und deren langfristi- & mationssystem & Patientenpfade \\
ge Auswirkungen & - Patientenpfade & - WP2. Metadaten für interorgani- \\
- Elektronische Fallakte & - Elektronische Fallakte & sationale psychotraumatologische \\
- IT-Standards der Medi- & - Systemarchitektur & Fallakten \\
zininformatik & - Schnittstellenspezifikation & - WP3. Methodik zur Entwicklung \\
- Organisationale Integra- & & interorganisationaler Fallakten via \\
tionsmodelle & & Fokusgruppen \\
- Qualitative Erhebungs- & & - WP4. CDA-Spezifikation des Psy- \\
ansätze für konsentierte & & chotraumatologischen Arztbriefs \\
Wissensbanken & & - WP5. Überführungskonzept \\
- Existierende & & FHIR-CDA \\
Diagnostik- & & - WP6. FHIR-Profilierung Psy- \\
Apps & chotraumatologie \\
& & - WP7. Referenzarchitektur \\
\hline & & \\
\hline & &
\end{tabular}

als Moderationshilfe und lenkten die Diskussionen zur erfolgreichen Identifikation impliziter Wissensbeiträge der DIP.

\subsection{Wissenstypen Digitaler Innovationsprojekte im Gesundheitswesen}

Anhand der Wissensbeiträge, die in den Fallstudien zusammengetragen wurden, wird im Folgenden eine Klassifikation abgeleitet. Dabei wurden die einzelnen Wissensbeiträge bezüglich vergleichbarer Eigenschaften klassifiziert und Wissenstypen zugeordnet. Die Wissenstypen sind dabei Spezialisierungen der Schlüsselkonzepte des Design Science: Konzepte, Modelle, Methoden und Instanziierungen (Drechsler and Hevner 2018). Im Ergebnis konnten sieben Wissenstypen klassifiziert werden. Dazu zählen technologische Architekturen, Spezifikationen, Digitale Versorgungsmodelle, Medizinische Fachmodelle, Integrationsmodelle, Gestaltungsempfehlungen und Methoden. Folgend werden diese Wissenstypen vorgestellt sowie die wesentlichen Charakteristika herausgearbeitet. Aufgrund ihrer Genese stehen die abgeleiteten Wissenstypen in direktem Bezug zur Domäne des Digital Health. Eine Adaption in andere Fachdomänen ist Gegenstand anschließender Forschung. Beispielsweise 
könnte der Wissenstyp „Digitale Versorgungsmodelle“ für die Privatwirtschaft durch einen Wissenstyp „Geschäftsmodelle“ substituiert werden.

Architekturen Architekturen sind ein wichtiger Wissenstyp von DIP. Sie beschreiben technologische Komponenten, systematisieren ihre Rollen und setzen diese zueinander in Beziehung. In Hinblick auf Integrationsaufgaben zeigen sie die zentralen Ansatzpunkte für Abstimmungsbedarf innerhalb eines Konsortiums auf und vermitteln Arbeitsaufgaben. Im Sinne eines Wissenstyps können Architekturen als Referenz für ähnliche Projekte dienen, insbesondere wenn damit Fragen des Datenschutzes, der Entkopplung von Komponenten und Verteilung von Arbeitsaufgaben vorgedacht sind. Typische Wissenschaftsgebiete zur Veröffentlichung von Architekturen sind die Informatik, Wirtschaftsinformatik und Medizinische Informatik. Hierbei bietet sich insbesondere das Forschungsgebiet der Referenzarchitekturen und Informationssystemmodellierung an.

Typisierte Wissensbeiträge: WS2, WN4, WP7.

Spezifikationen Spezifikationen beschreiben in detaillierter Form, wie konkrete Systembestandteile einer Architektur ausgestaltet sein sollten. Sie verlassen Betrachtungen von Architekturen und wenden sich dem Entwurf einer konkreten Lösung zu. Eine Spezifikation kann im Gesundheitswesen sowohl fachbezogen (z. B. Spezifikation eines digitalen Schlaganfallpasses) als auch technologisch (z.B. FHIRProfil zum Austausch von Fragebögen) beschrieben werden, wobei die Grenzen fließend sind. Häufig sind Spezifikationen im Bereich Digital Health mit dieser Aufgabe konfrontiert, eine adäquate Balance zwischen medizinischen Fachmodellen und technologischen Detaillierungen zu finden. Die Veröffentlichung von Spezifikationen kann im Kontext von Standardisierungsgremien im Bereich der Medizinischen Informatik erfolgen. Auch wird die konkrete Implementierung von Standards im Kontext von Fallstudien in diesem Bereich veröffentlicht.

Typisierte Wissensbeiträge: WS1, WN2, WP4, WP6.

Digitale Versorgungsmodelle Ein Digitales Versorgungsmodell beschreibt ähnlich den Geschäftsmodellen in der Privatwirtschaft die zentralen und logischen Funktionsweisen innerhalb eines definierten Leistungsgeflechts von Akteuren. Typischerweise beschreiben sie eine idealisierte und koordinierte Versorgung für den Patienten über verschiedene Leistungserbringer hinweg. Digitale Versorgungsmodelle heben hierbei die Verwendung von digitaler Technologie zur Leistungserbringung hervor, fassen das Leistungsgeflecht und Leistungsversprechen sowie die zentralen Geschäftsprozesse zusammen. Im Kontext der vorgestellten Fallstudien werden drei verschiedene Versorgungsmodelle abgebildet. Die typischen Forschungsgebiete zur Veröffentlichung von Versorgungsmodellen finden sich im Bereich der Versorgungsforschung, im Bereich von Public Health sowie in der Gesundheitsökonomie und der gesundheitsorientierten Betriebswirtschaftslehre.

Typisierte Wissensbeiträge: WS4, WN3.

Medizinische Fachmodelle Medizinische Fachmodelle beschreiben strukturelle (z. B. semantische Repräsentationen) oder dynamische (z. B. Patientenpfade) Aspek- 
te des Versorgungsszenarios. Sie dienen als visuelle und zugleich (semi-)formale Repräsentation medizinischer Sachverhalte. Sie können sowohl durch Fachexperten zum Aufbau eigener Versorgungsmodelle als auch durch IT-Spezialisten zur Herleitung von Spezifikationen verwendet werden. Medizinische Fachmodelle können im Bereich der Medizin bzw. der medizinischen Dokumentation als auch in der medizinischen Informatik veröffentlicht.

Typisierte Wissensbeiträge: WP1, WP2.

Integrationsmodelle Integrationsmodelle beschreiben sowohl methodisch als auch strukturell, wie Partner im Bereich der Digitalen Versorgungsmodelle miteinander fachliche als auch technologische Zusammenarbeit praktizieren können und wie diese Zusammenarbeit initiiert werden kann. Die Veröffentlichung solcher Wissensbeiträge kann im Teilgebiet Enterprise Integration im Wissenschaftsbereich der Wirtschaftsinformatik, in der Medizinischen Informatik sowie in der Versorgungsforschung erfolgen.

Typisierte Wissensbeiträge: WN5, WN3.

Gestaltungsempfehlungen Gestaltungsempfehlungen können sowohl Digitale Versorgungsmodelle als auch medizinische Technologien adressieren. Sie leiten aus dem Kontext eines solchen Modells bzw. einer solchen Technologie Empfehlungen für die fachliche und technische Ausgestaltung ab. Dabei berücksichtigen sie das organisationale Umfeld und beteiligte Stakeholder (z.B. User InterfaceNutzungsprinzipien für bestimmte Patientenkohorten). Sie können auch aus konkreten Implementierungen resultieren. Je nach Ausprägung lassen sich diese in allen Bereichen des Gesundheitswesens als auch der Informatik einbringen.

Typisierte Wissensbeiträge: WN1, WN6, WP5.

Methoden Im Zuge von Projekten im Bereich der Digitalen Innovation werden verschiedene Vorgehen zur Entwicklung neuer Artefakte eingesetzt (z.B. Anwendung von Fokusgruppen zur Erhebung einer konsentierten einrichtungsübergreifenden Aktenstruktur). Die Formalisierung und Generalisierung dieser methodischen Herangehensweisen zu einem wiederverwendbaren Wissensbeitrag können durch Digital Health Projekte adressiert werden. Solche Methoden können sowohl in der Wirtschaftsinformatik, Medizinischen Informatik als auch der Versorgungsforschung veröffentlicht werden.

Typisierte Wissensbeiträge: WS3, WN7, WP3.

\section{Kritische Würdigung und Ausblick}

Im vorliegenden Beitrag wurde untersucht, welche Konzepte und Ansätze der aktuelle wissenschaftliche Diskurs zur Systematisierung von Wissensbeiträgen in gestaltungsorientierten Projekten bereithält. Hierfür wurden zunächst das Design Science Research-Grids nach vom Brocke and Maedche (2019) eingeführt und die Rolle von deskriptivem und präskriptivem Wissen innerhalb der Forschung erörtert. Da Praxisprojekten oftmals die Struktur fehlt, ihre Innovation(en) und Resultate in ge- 
eigneter Form in generalisierbares präskriptives Wissen zu überführen, bietet dieses Grid einen guten Moderationsrahmen zur Analyse und Explikation von Wissen innerhalb solcher Praxisprojekte. Dies bedarf aber ebenso Sensibilität innerhalb der Projektorganisation für eine frühzeitiges Erheben der Informationen sowie der notwendigen Kommunikation innerhalb des Projektkonsortiums.

Die durchgeführte Fallstudie illustriert anhand von drei DIP, wie eine solche Systematisierung aussehen kann. Sie zeigt aber auch auf, dass insbesondere die Generalisierung der Artefakttypen insoweit herausfordernd ist, als dass die Innovationshöhe eines DIP nicht automatisch aus der Grid-basierten Beschreibung hervorgeht und sich im Vergleich zum Problem-Lösungsraum weniger gut darstellen lässt.

Die Explikation von präskriptiven Gestaltungswissen wird jedoch wesentlich aufgewertet und in eine einheitliche Struktur gebracht, sodass dieses leichter von ähnlichen Projektkonstellationen als Input(wissen) verwendet werden kann. Gleichzeitig verbindet sich damit die Herausforderung, dass zur Kommunikation des Wissensbeitrags (Output) generalisierte Wissenstypen genutzt werden sollten. Dadurch können die Lösungen auch für problemähnliche Konstellationen einen Beitrag liefern. Die vorgestellten Wissenstypen können als Konkretisierungen klassischer Artefakttypen innerhalb der gestaltungsorientierten Forschung betrachtet werden. Ihre Reichweite und Gültigkeit sind aufgrund ihrer induktiven Ableitung limitiert auf die zugrundeliegenden Kontexte des Forschungs- und Entwicklungsbereichs Digital Health. Bei den vorgestellten Wissenstypen handelt es sich somit um keine abgeschlossene Menge. Vielmehr zeigt der Beitrag, wie entstehendes Wissen systematisiert werden kann und wie ein entsprechender Wissenstyp gekennzeichnet ist. Wenngleich die Wissenstypen induktiv entstanden sind, können sie doch als Referenz und Legitimation für Forschungsziele dienen.

Aus wissenschaftlicher Sicht können die Wissenstypen außerdem dazu beitragen, Digital Health-Projekte zu klassifizieren. Mit dem Tupel von Problemraum, Wissenstyp und angewandtem Versorgungsmodell können typische Archetypen verbunden werden, welche wiederum als Muster für ähnliche Projekte herangezogen werden können. Ebenfalls ist ihre Nutzung zur Evaluation von bestehenden Implementierungen möglich.

In anschließenden Untersuchungen sollte der vorgeschlagene Ansatz weiter erprobt und seitens der Fachanwender kritisch evaluiert werden. Hierbei sollte insbesondere untersucht werden, innerhalb welcher Phasen eines Umsetzungsprojektes bereits welche Informationen aufzunehmen sind. Unabhängig davon sollte auch analysiert werden, wie bestehende Entwicklungsmethoden z. B. Agile Softwareentwicklung mit dem Anspruch einer systematischen Wissensexplikation in Einklang gebracht werden können.

Funding Open Access funding provided by Projekt DEAL.

Open Access Dieser Artikel wird unter der Creative Commons Namensnennung 4.0 International Lizenz veröffentlicht, welche die Nutzung, Vervielfältigung, Bearbeitung, Verbreitung und Wiedergabe in jeglichem Medium und Format erlaubt, sofern Sie den/die ursprünglichen Autor(en) und die Quelle ordnungsgemäß nennen, einen Link zur Creative Commons Lizenz beifügen und angeben, ob Änderungen vorgenommen wurden. 
Die in diesem Artikel enthaltenen Bilder und sonstiges Drittmaterial unterliegen ebenfalls der genannten Creative Commons Lizenz, sofern sich aus der Abbildungslegende nichts anderes ergibt. Sofern das betreffende Material nicht unter der genannten Creative Commons Lizenz steht und die betreffende Handlung nicht nach gesetzlichen Vorschriften erlaubt ist, ist für die oben aufgeführten Weiterverwendungen des Materials die Einwilligung des jeweiligen Rechteinhabers einzuholen.

Weitere Details zur Lizenz entnehmen Sie bitte der Lizenzinformation auf http://creativecommons.org/ licenses/by/4.0/deed.de.

\section{Literatur}

Becker J, Holten R, Knackstedt R, Niehaves B (2003) Forschungsmethodische Positionierung in der Wirtschaftsinformatik: epistemologische, ontologische und linguistische Leitfragen. Arbeitsberichte des Instituts für Wirtschaftsinformatik. Westfälische Wilhelms-Universität Münster, Münster

Drechsler A, Hevner AR (2018) Utilizing, producing, and contributing design knowledge in DSR projects. In: Chatterjee S, Dutta K, Sundarraj RP (Hrsg) Designing for a digital and globalized world. Springer, Cham, S 82-97

Fichman RG, Dos Santos BL, Zheng Z (2014) Digital innovation as a fundamental and powerful concept in the information systems curriculum. MISQ 38:329-343

Frank U (1998) The MEMO meta-metamodel

Herrmann M, Boehme P, Mondritzki T et al (2018) Digital transformation and disruption of the health care sector: Internet-based observational study. J Med Internet Res 20:e104. https://doi.org/10.2196/jmir. 9498

Hevner A, vom Brocke J, Maedche A (2019) Roles of digital innovation in design science research. Bus Inf Syst Eng 61:3-8. https://doi.org/10.1007/s12599-018-0571-z

Laurenza E, Quintano M, Schiavone F, Vrontis D (2018) The effect of digital technologies adoption in healthcare industry: a case based analysis. Business Process Mgmt Journal 24:1124-1144. https:// doi.org/10.1108/BPMJ-04-2017-0084

March ST, Smith GF (1995) Design and natural science research on information technology. Decis Support Syst 15:251-266. https://doi.org/10.1016/0167-9236(94)00041-2

Matusiewicz D, Pittelkau C, Elmer A, Addam M (Hrsg) (2017) Die digitale Transformation im Gesundheitswesen: Transformation, Innovation, Disruption. Medizinisch Wissenschaftliche Verlagsgesellschaft, Berlin

Österle H, Becker J, Frank U et al (2011) Memorandum on design-oriented information systems research. Eur J Inf Syst 20:7-10. https://doi.org/10.1057/ejis.2010.55

Otto B, Österle H (2010) Relevance through consortium research? Findings from an expert interview study. In: Winter R, Zhao JL, Aier S (Hrsg) Global perspectives on design science research. Springer, Berlin, Heidelberg, S 16-30

Venable J, Pries-Heje J, Baskerville R (2012) A comprehensive framework for evaluation in design science research. In: Design science research in information systems. Advances in theory and practice. Springer, Berlin Heidelberg, S 423-438

vom Brocke J, Maedche A (2019) The DSR grid: six core dimensions for effective capturing of DSR projects

Werner M (2019) Exploring design science research project characteristics—an initial empirical investigation. In: HICSS 2019 proceedings, S 5705-5714

Yoo Y, Henfridsson O, Lyytinen K (2010) The new organizing logic of digital innovation: an agenda for information systems research. Inf Syst Res 21:724-735. https://doi.org/10.1287/isre.1100.0322 